\title{
Prediction of complications in inflammatory bowel disease using routine blood parameters at diagnosis
}

\author{
Tong $\mathrm{Li}^{1,2 \#}$, Yuting Qian ${ }^{1 \#}$, Tingting Bai ${ }^{1}$, Juanjuan $\mathrm{Li}^{1,2}$ \\ ${ }^{1}$ Department of Medical Oncology, Shanghai Pulmonary Hospital \& Thoracic Cancer Institute, Tongji University School of Medicine, Shanghai, \\ China; ${ }^{2}$ Department of Gastroenterology, Ruijin Hospital Affiliated to Shanghai Jiao Tong University School of Medicine, Shanghai, China \\ Contributions: (I) Conception and design: T Li, J Li; (II) Administrative support: J Li; (III) Provision of study materials or patients: T Li, Y Qian, T \\ Bai; (IV) Collection and assembly of data: T Li, Y Qian; (V) Data analysis and interpretation: T Li, J Li; (VI) Manuscript writing: All authors; (VII) \\ Final approval of manuscript: All authors. \\ "These authors contributed equally to this work. \\ Correspondence to: Juanjuan Li, MD, PhD. Department of Medical Oncology, Shanghai Pulmonary Hospital \& Thoracic Cancer Institute, Tongji \\ University School of Medicine, 507 Zheng Min Road, Shanghai 200433, China. Email: lijuanjuan700@126.com.
}

Background: Patients with inflammatory bowel disease (IBD) can develop a series of complications over time, including fistulas and perforation, strictures and pseudopolyp formation. Whether there are some effective and economical routine inspection indicators for the early prediction and prevention of complications has clinical significance and is worth exploring.

Methods: A total of 592 newly diagnosed IBD patients [153 with ulcerative colitis (UC), 439 with Crohn's disease $(\mathrm{CD})$ ] were enrolled in a retrospective study. We compared the baseline characteristics (age at diagnosis, sex, clinical disease location and stage), serum C-reactive protein (CRP) level, serum erythrocyte sedimentation rate (ESR) level, total white blood cell (WBC), eosinophil (EOS) and platelet (PLT) counts for all IBD patients. And we evaluated the correlation between these routine parameters and the occurrence of clinical complications (including fistula, perforation, stricture, pseudopolyps or cancerous changes) in the course of IBD by univariate and multivariate analyses. Moreover, we constructed a dual parameter prediction system for complication's verification.

Results: We demonstrated that higher serum CRP levels and total WBC counts independently indicated a higher hazard for the earlier occurrence of pseudopolyp formation in UC patients. Likewise, higher serum ESR and lower eosinophil count predicted the development of fistulas in CD patients, and higher serum ESR and PLT counts accounted for CD patients' strictures.

Conclusions: Novel predictive models including CRP level, ESR, WBC count, PLT and eosinophils were structured to predict complications in IBD, which might enable more aggressive treatment for patients at risk.

Keywords: Prediction; complication; inflammatory bowel disease (IBD); routine blood parameters

Submitted Dec 17, 2021. Accepted for publication Feb 16, 2022.

doi: $10.21037 / \mathrm{atm}-22-123$

View this article at: https://dx.doi.org/10.21037/atm-22-123

\section{Introduction}

Asia, where some countries are undergoing rapid urbaniszation and adoption of an industrialized lifestyle, is witnessing an increase in the annual incidence of inflammatory bowel disease (IBD), including both ulcerative colitis (UC) and Crohn's disease (CD). The severity of the course of IBD is highly variable, with some patients developing devastating complications and a sizeable minority experiencing an indolent course (1-3). The main complications of CD include intestinal obstruction, colon cancer, fistula and etc. And perforation, fistula, cancerous 
changes, pseudopolyp formation, toxic megacolon and stenosis disease was defined as the main complication of UC. Complications of IBD can lead to shock, the need for abdominal surgery, or even sudden death. The ultimate goal of medical therapy in IBD, beyond achieving clinical response and sustained remission, is to alter the natural history of the disease; that is, slowing or preventing the occurrence of complications is of great importance (4).

Disease progression and complications could be affected by recent advances in early intervention with immunosuppressant and biological anti-inflammatory therapies $(5,6)$. However, the treatment of all patients with biologics and/or combination therapy is economically unsustainable and would risk exposing those with an indolent disease course to unnecessary risks or side effects of potent therapy. Currently, there is a paucity of data that can guide clinicians dealing with this condition (7). Therefore, the challenge we face lies in the development of diagnostic tools to identify patients who are at risk of developing a complicated disease course and who will be appropriate candidates for more aggressive therapy.

IBD is a polygenic disorder with hundreds of risk loci identified by numerous genome-wide association studies (GWASs), led by the discovery of NOD2, and more than 200 susceptibility genes have been identified so far (8-10). Given the wide range of phenotypes and the diverse spectrum of disease behaviour within the conditions we currently label CD and UC, it is becoming increasingly clear that different biomarkers and phenotypic characteristics at IBD diagnosis could assist in greater predictive ability (11-16). However, these genetic contributions together likely explain only $19-26 \%$ of the hereditary variance of IBD (17), and the risk of disease development or the expression of a specific phenotype in a CD/UC patient are not predicted or understood solely by genetic risk. Additionally, many of these tests are investigational, expensive and not routinely available. Importantly, in addition to unravelling novel aspects of disease pathogenesis, we urgently require novel clinical signatures to allow better classification of clinically relevant disease entities. Complex interactions among susceptibility genes and the environment could lead to inappropriate immune activation with an infiltrate of immune cells and a severe massive production of proinflammatory cytokines contributes to the development of IBD, which may cause severe repeating local tissue damage and triggered the onset of different complications. Studies have shown that WBC count, CRP and ESR could reflect the activity of colon lesions and the severity of disease, as well as currently known to associate with immune function (18-20). Could these parameters also be predictors of IBD complications?

In our current study, we used the above indicators present at the diagnosis of CD and UC to predict the occurrence of complications rather than the severity of disease, and we furtherly classified white blood cells, and combined with significant independent predictors to further refine the prediction model in Chinese IBD patients. We confirmed that instead of costly examination techniques and expensive biomarkers, these simple parameters could be an economical predictor of IBD complications. We present the following article in accordance with the STROBE reporting checklist (available at https://atm.amegroups.com/article/ view/10.21037/atm-22-123/rc).

\section{Methods}

\section{Study population criteria}

We performed a single-centre retrospective cohort study of untreated IBD patients who were hospitalised at Ruijin Hospital Affiliated to Shanghai Jiao Tong University School of Medicine between January 2012 and June 2018 who were able to undergo total colonoscopy, and have a good compliance with disease treatment, available for new biologics. Patients with unclassified IBD were excluded, as were patients with incomplete information in clinical records, laboratory tests or with obvious cachexia. All cases were diagnosed based on clinical presentation and radiological, endoscopic and pathological criteria by an expert gastroenterologist $(1,2)$. Follow-up was conducted by hospitalization and telephone contact. All procedures performed in this study involving human participants were in accordance with the Declaration of Helsinki (as revised in 2013). The study was approved by the Ethics Committee at Ruijin Hospital Affiliated to Shanghai Jiao Tong University School of Medicine (approval ID: 2014-Clinical Ethics Review No.16a). Individual consent for this retrospective analysis was waived.

A complication of $C D$ was defined as one of the following: penetrating disease, stricturing disease, perianal manifestation or abdominal surgery. A complication of $\mathrm{UC}$ was defined as: perforation, fistula, cancerous changes, pseudopolyp formation or stenosis. Patients with any complications on first diagnosis were excluded. Within the 6-year period reviewed, 648 consecutive patients were first diagnosed with IBD at Ruijin Hospital Affiliated to 
Shanghai Jiao Tong University School of Medicine. Of them, 56 were excluded because of incomplete data. In total, 592 newly diagnosed patients, comprising 153 UC patients and $439 \mathrm{CD}$ patients, were included in this study.

\section{Phenotype data collection and optimal cut-off settings}

The following clinical factors were recorded on the first day of initial hospitalisation: sex, age, routine blood parameters, serum ESR, serum CRP level, lesion site and disease severity. The severity of disease was assessed using the Lichtiger score for patients with UC, and the Crohn's Disease Activity Index for patients with CD. As there are no established criteria for using routine data for complication prediction in IBD, we next determined the optimal cut-off values based on receiver operating characteristic (ROC) analysis. This curve is drawn using sensitivity ( $t$ ) and 1 - specificity $(t)$, which are obtained from various cut-off values of a biomarker a time t. By using these measurements, a time-dependent ROC curve can be drawn at any time. The Youden index method was used to calculate optimal cut-off values.

\section{Statistical analysis}

We used SPSS version 22.0 for all statistical analyses (IBM Corp., Armonk, NY, USA). Descriptive statistics included the calculation of median values (range) for all continuous variables. The categorical variables were summarised using absolute frequencies and percentages and compared with the Chi-square test. We used Pearson's test for bivariate correlational analysis and one-way analysis of variance test for means and standard deviations (SD), with the interquartile range representing continuous variables. Univariate analysis (with logistic regression) was performed to identify the variables that were associated with complications of IBD. Risk factors were identified after the univariate analysis. Next, multivariate analysis with logistic regression and a forward likelihood ratio test was performed to determine independent risk factors closely associated with the incidence of complications. All the independent variables with significance in the univariate analysis were introduced in stepwise manner to the multivariate analysis to identify predictors of IBD. Finally, the predicted probability for IBD complications was used for the evaluation of predictive ability. $\mathrm{P}<0.05$ was considered significant in this study.

\section{Results}

\section{Patients' characteristics and clinical complications}

Within the 6-year period reviewed, 648 consecutive patients were first diagnosed with IBD at Ruijin Hospital Affiliated to Shanghai Jiao Tong University School of Medicine. Of them, 56 were excluded because of incomplete data, poor treatment compliance or obvious cachexia. In total, 592 newly diagnosed IBD patients (153 with UC, 439 with CD) were included, with a median follow-up time of 69.6 months. There were 319 male patients $(72.7 \%)$ and $120(27.3 \%)$ female patients with CD, and 99 (64.7\%) and $54(35.3 \%)$ respectively with UC. In general, the mean age at hospitalization was $34.19 \pm 11.73$ years in CD patients, and $45.93 \pm 15.6$ years in UC patients. Lesion location, severity and routine laboratory data on admission [i.e., white blood cell (WBC) count, haemoglobin (Hb), platelet (PLT) count, serum CRP level and ESR] are listed in Table 1.

During the observation period, most patients were hospitalized multiple times for additional treatment. As shown in Table 1, for CD patients, the incidence for strictures was $330 / 439$ (75.1\%), 124/439 (28.2\%) for fistulas, $17 / 439$ (3.87\%) for perforations, and 68/439 (15.5\%) for abdominal surgery. For UC patients, the incidence for pseudopolyp formation was 59/153 (38.5\%), $21 / 153(13.7 \%)$ for strictures, $3 / 153(1.96 \%)$ for fistulas, $1 / 153(0.65 \%)$ for cancerous change, and $11 / 153$ (7.1\%) for abdominal surgery rate. None of the patients died as a result of IBD.

\section{Differences in routine laboratory parameters between $C D$ and UC patients}

Because the pathogenesis and mechanism of UC and CD are not exactly the same, to explore whether there were any differences between them in the routine laboratory data, we first analysed the WBC counts, Hb levels, PLT counts, serum CRP levels and ESR on admission for the CD and UC patients. As shown in Figure 1, the WBC count of UC patients was significantly higher than that of $C D$ patients $(\mathrm{P}<0.0001)$, specifically manifested as increased lymphocytes $(\mathrm{P}<0.0001)$, neutrophils $(\mathrm{P}<0.0001)$ and monocytes $(\mathrm{P}=0.0248)$. In addition, the CRP level in the UC group was also increased significantly compared with that in the $\mathrm{CD}$ group $(\mathrm{P}=0.0369)$.

IBD is characterised by an intestinal immune cell infiltrate, and gut homing of lymphocytes has been 
Table 1 Baseline characteristics of patients and laboratory data on admission

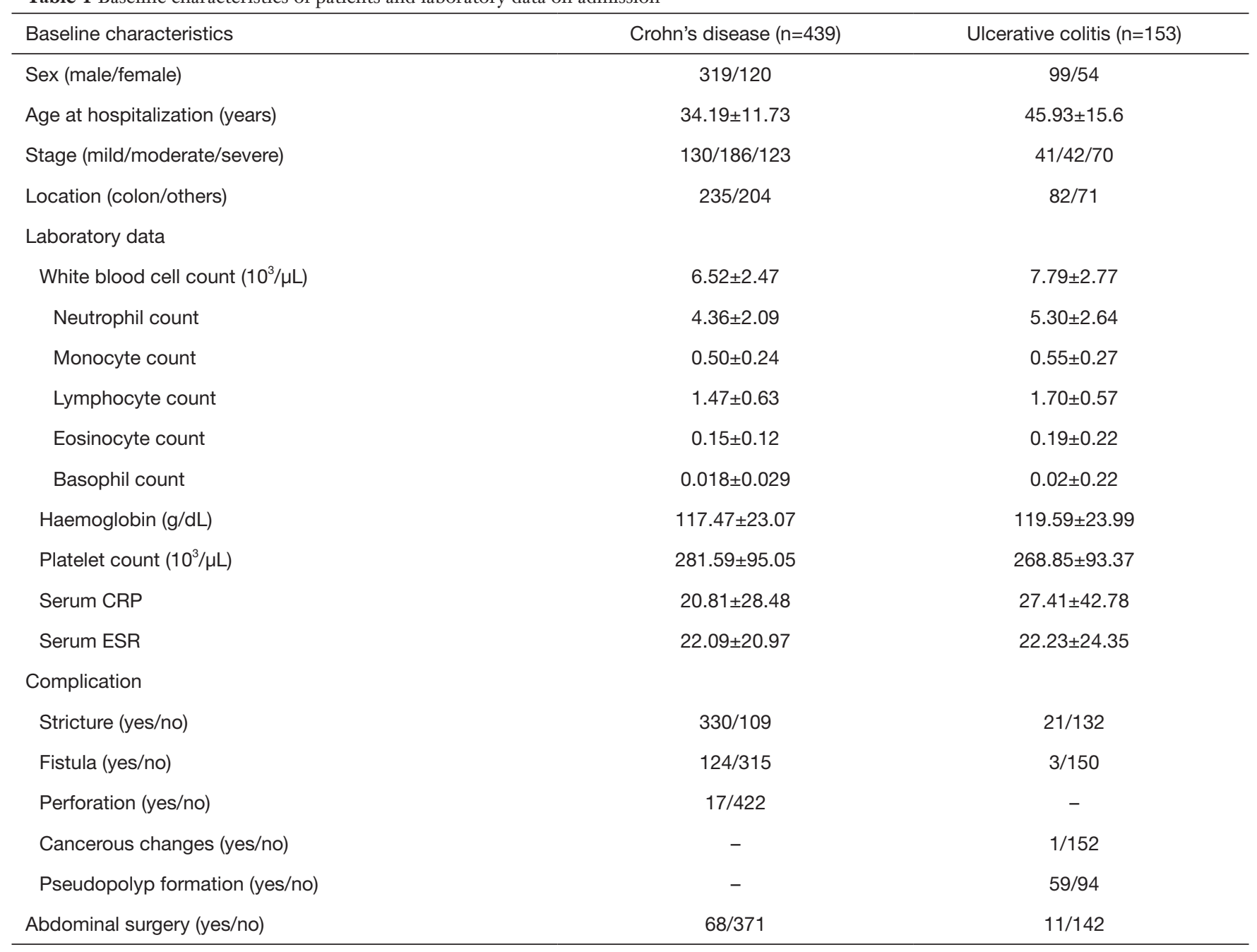

Data are expressed as means $\pm \mathrm{SD}$. CRP, C-reactive protein; ESR, serum erythrocyte sedimentation rate.

recognised as a promising target for therapy. A variety of approaches targeting leukocyte trafficking have been studied, with variable success $(21,22)$. Anti-integrin therapy blocks the action of integrin on the surface of circulating immune cells and endothelial cell adhesion molecules, thereby inhibiting the interactions between leukocytes and intestinal blood vessels. For instance, vedolizumab, a humanised anti- $\alpha 4 \beta 7$-integrin antibody, has demonstrated efficacy in patients with IBD and has an excellent safety profile (23-25). Inspired by the differences between the UC and $\mathrm{CD}$ groups in terms of leukocytes, it may be possible to further refine the method of leukocyte trafficking treatment in the future to obtain the best curative effect.

\section{Comparison of complication and non-complication groups of CD patients}

We analysed the routine parameters between CD patients with and without fistula, perforation or stricture. On admission, patients with fistula had a higher serum ESR $(\mathrm{P}=0.0221)$ and a lower eosinophil count $(\mathrm{P}=0.0121)$ (Figure 2A). Similarly, patients with a higher ESR had a higher rate of fistula incidence $(\mathrm{P}=0.002)$, and a higher eosinophil count may have a protective effect on fistula development $(\mathrm{P} \leq 0.001)$ (Figure $2 B)$. For patients with stricture complications, serum ESR and PLT count were associated with their occurrence $(\mathrm{P}=0.0322$ and 0.0089 , 

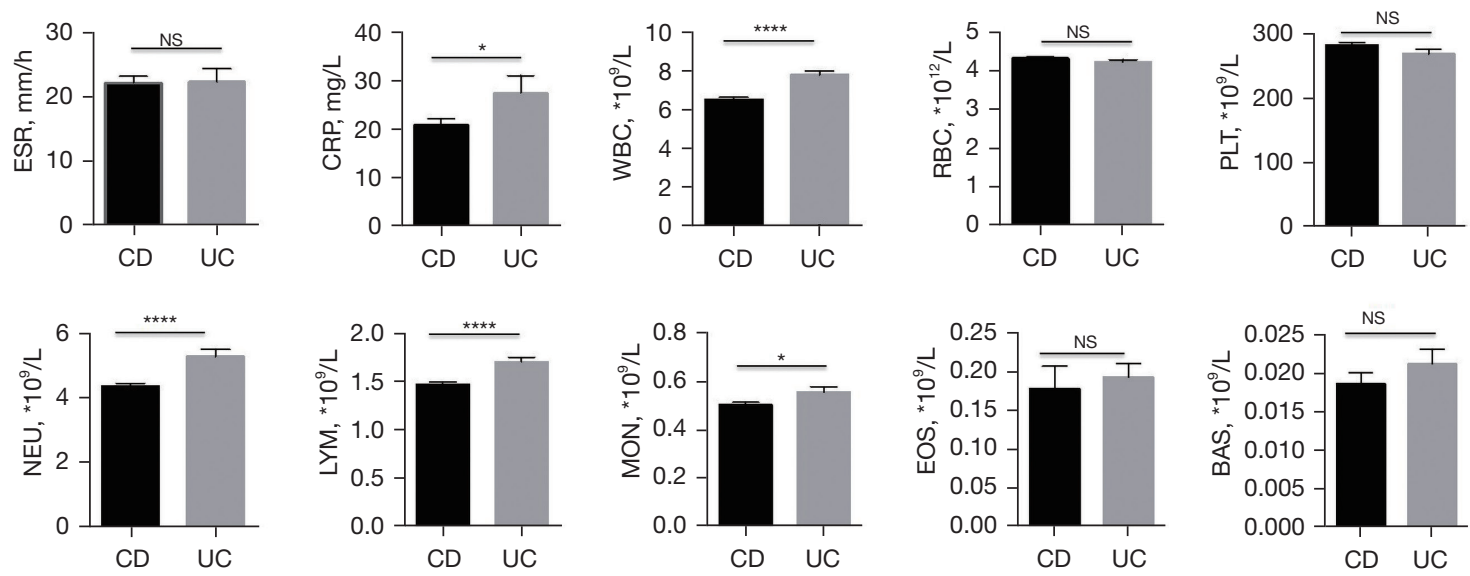

Figure 1 Differences in routine laboratory parameters between CD and UC patients. Differences in serum ESR and CRP level, WBC count (including lymphocytes, neutrophils, monocytes, eosinophils and basophil), RBC count, and PLT count, on admission between CD and UC patients. *, $\mathrm{P}<0.05$; **** $\mathrm{P}<0.0001$. CD, Crohn's disease; UC, ulcerative colitis; CRP, C-reactive protein; ESR, erythrocyte sedimentation rate; WBC, white blood cell; RBC, red blood cell; PLT, platelet; NS, not significance.

A
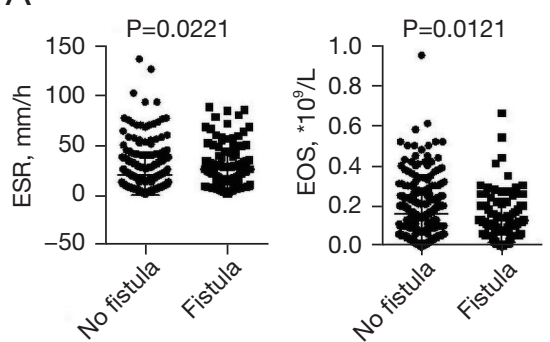

C

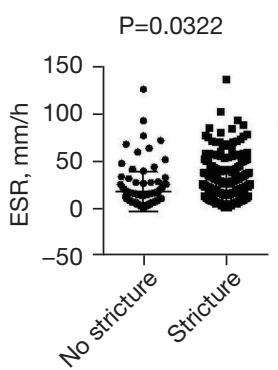

B
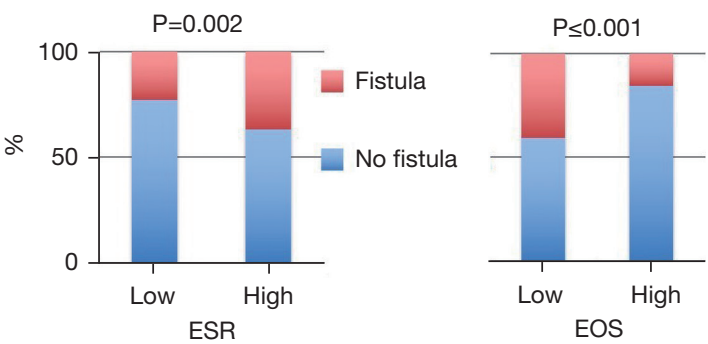

D

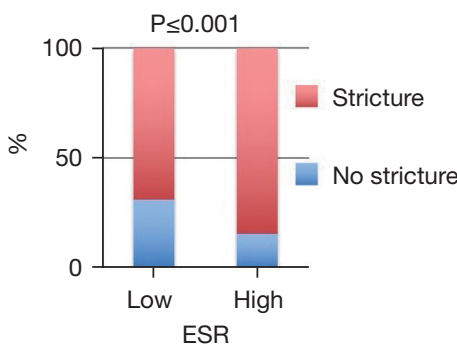

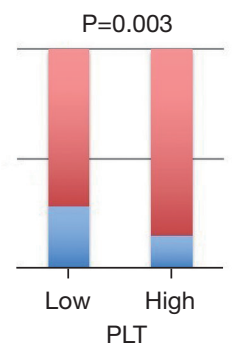

Figure 2 Comparison of complication and non-complication groups of CD patients. (A) Patients with fistulas had a higher serum ESR ( $\mathrm{P}=0.0221)$ and a lower eosinophil count compared with patients without fistulas $(\mathrm{P}=0.0121)$; (B) patients with a higher ESR level or lower eosinophil count had a higher rate of fistula incidence ( $\mathrm{P}=0.002$ and $\mathrm{P} \leq 0.001$, respectively); (C) patients with strictures had a higher serum ESR ( $\mathrm{P}=0.0322)$ and PLT count $(\mathrm{P}=0.0089)$ compared with patients without strictures; (D) association of increased serum ESR and PLT count and the occurrence of strictures ( $\mathrm{P} \leq 0.001$ and 0.003 , respectively). CD, Crohn's disease; ESR, erythrocyte sedimentation rate; PLT, platelet. 
A

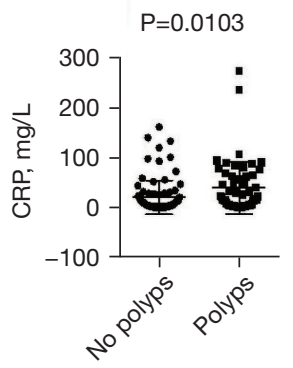

C

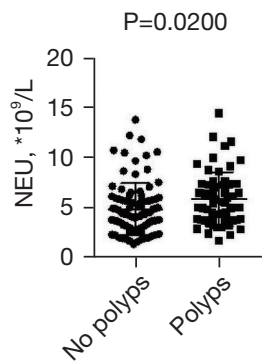

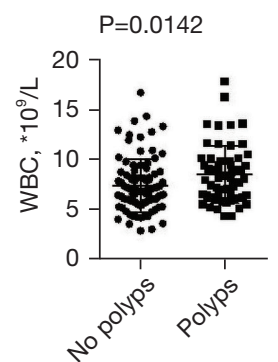

B
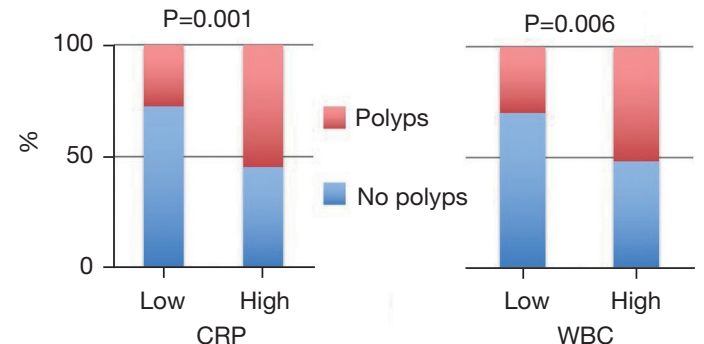

Figure 3 Comparison of pseudopolyp and non-pseudopolyp groups of UC patients. (A) Serum CRP levels, as well as WBC counts were greatly increased in patients with pseudopolyps compared with those without pseudopolyps ( $\mathrm{P}=0.0103$ and 0.0142 , respectively); (B) association of higher serum CRP levels and WBC counts with higher rates of pseudopolyp formation ( $\mathrm{P}=0.001$ and 0.006 , respectively); (C) analysis of different types of WBCs showed that neutrophil counts were greatly increased in patients with pseudopolyps ( $\mathrm{P}=0.02)$, and the same was true for monocytes ( $\mathrm{P}=0.0251)$. UC, ulcerative colitis; $\mathrm{CRP}$, C-reactive protein; WBC, white blood cell.

respectively) (Figure 2C). Likewise, when we conducted stratification analysis according to ESR and PLT level, we also concluded that their increase could predict the occurrence of complications $(\mathrm{P} \leq 0.001$ and 0.003 , respectively) (Figure $2 D$ ). There were no significant differences in serum CRP level, HB, haematocrit, or other WBC counts on admission between the complication and non-complication groups of CD patients.

\section{Comparison of pseudopolyp and non-pseudopolyp groups of UC patients}

Pseudopolyps, as the most common complication in UC patients, can be an indirect marker of repeated episodes of severe inflammation and excessive healing processes, and their incidence rises with more extensive colitis (26). Thus, it would be helpful to know which indicators can predict pseudopolyp formation. In our study, we found that higher serum CRP level and WBC counts were associated with higher pseudopolyp formation rates $(\mathrm{P}=0.0103$ and 0.0142 , respectively) (Figure $3 A$ ); moreover, similar results were obtained when stratified grouping was conducted according to the level of serum CRP and WBC count $(\mathrm{P}=0.001$ and 0.006 , respectively) (Figure $3 B$ ). In addition, analysis of the relationship with other routine parameters showed that neutrophil counts were greatly increased in patients with pseudopolyps $(\mathrm{P}=0.0200)$, and the same was true for monocyte counts $(\mathrm{P}=0.0251)$ (Figure 3C). We also investigated the occurrence of intestinal stricture, but unfortunately, no valuable predictors were found. Other complications, including fistula, perforation, and cancerous changes, were rare, and no further analysis was performed.

\section{Univariate and multivariate analysis for predictors of complications in patients with UC/CD}

To further evaluate the predictive ability of the markers identified above, we conducted univariate and multivariate logistic regression analyses and considered potential confounders: age at diagnosis, sex, clinical disease location and stage for all IBD patients, adding serum CRP level and WBC count for UC patients, and serum ESR and eosinophil and PLT counts for CD patients.

As shown in Table 2, stage, serum CRP level and WBC 
Table 2 Predictive factors for intestinal polyps of colitis in univariate and multivariate logistic regression analyses

\begin{tabular}{|c|c|c|c|c|c|c|c|c|}
\hline Variable & Category & $\begin{array}{l}\text { Patient } \\
\text { number }\end{array}$ & \multicolumn{3}{|c|}{ Univariate analysis } & \multicolumn{3}{|c|}{ Multivariate analysis } \\
\hline Age & $<40 / \geq 40$ y & $57 / 96$ & 0.619 & $0.311-1.234$ & 0.173 & 0.527 & $0.247-1.125$ & 0.098 \\
\hline Sex & Male/female & $99 / 54$ & 0.707 & $0.354-1.414$ & 0.327 & 0.625 & $0.295-1.322$ & 0.219 \\
\hline Stage & $<6 / \geq 6$ points & $83 / 70$ & 0.511 & $0.264-0.989$ & $0.046^{\star}$ & 0.733 & $0.350-1.537$ & 0.411 \\
\hline CRP & $<12.85 / \geq 12.85$ & $91 / 62$ & 0.312 & $0.158-0.616$ & $0.001^{*}$ & 0.365 & $0.170-0.782$ & $0.010^{*}$ \\
\hline WBC & $<7.895 \times 10^{9} / \geq 7.895 \times 10^{9}$ & $91 / 62$ & 0.396 & $0.202-0.774$ & $0.007^{*}$ & 0.507 & $0.243-1.058$ & 0.070 \\
\hline
\end{tabular}

*, $\mathrm{P}<0.05$ was considered significant. $\mathrm{P}$ values were calculated by univariate and multivariate binary logistic regression analysis. $\mathrm{Cl}$, confidence interval; OR, odds ratio; CRP, C-reactive protein; WBC, white blood cell.

Table 3 Predictive factors for fistula in Crohn's disease in univariate and multivariate logistic regression analyses

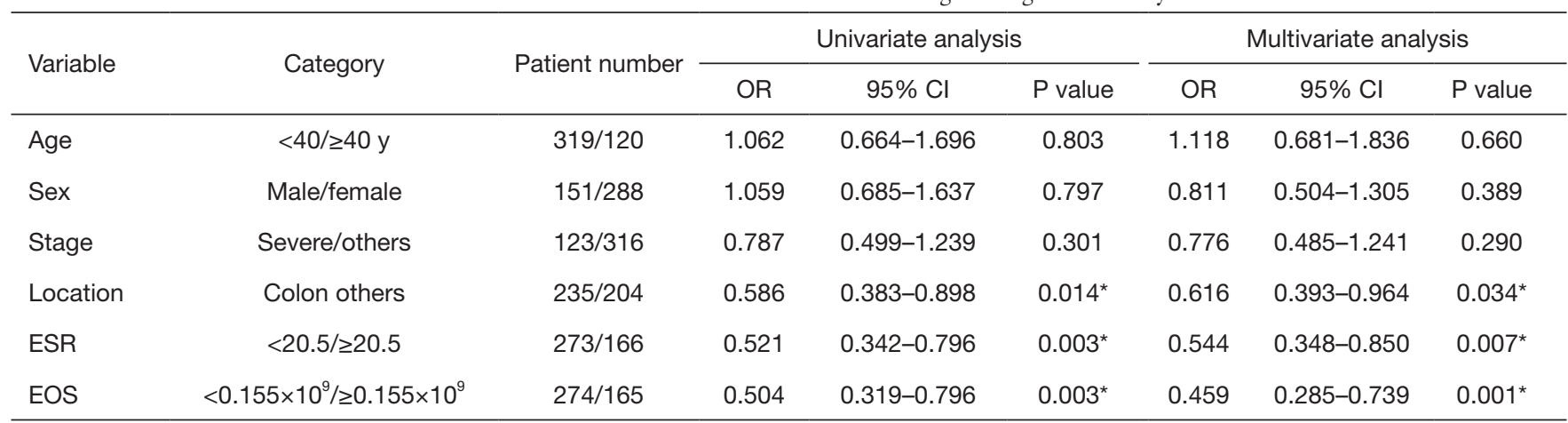

*, $\mathrm{P}<0.05$ was considered significant. $\mathrm{P}$ values were calculated by univariate and multivariate binary logistic regression analysis. Cl, confidence interval; OR, odds ratio; ESR, serum erythrocyte sedimentation rate; EOS, eosinophil.

count were associated with UC patients' pseudopolyp formation in univariate analysis. After adjusting the prognostic covariates by multivariate Cox regression, a higher serum CRP level continued to be associated with pseudopolyp formation, and this was independent of disease stage $(\mathrm{P}=0.01)$. With regard to $\mathrm{CD}$ patients' complications, lesion location, serum ESR and eosinophil level could predict fistula; in addition, older age, more severe lesions, and higher ESR and PLT count could indicate stricture, which were all true in both univariate and multivariate analyses (Tables 3,4).

\section{Dual parameter prediction system verification}

Based on our findings, we had a preliminary indication that blood tests routinely used for IBD patients in the clinical setting could act as predictors of complications; that is, higher serum CRP levels and WBC counts were associated with pseudopolyp formation in UC patients, whereas in CD patients, higher serum ESR and lower eosinophil counts were associated with fistula, and higher serum ESR and PLT counts were associated with stricture. To optimise the predictive capability, we constructed united predictive models. As shown in Figure 4A, patients with high CRP level and WBC count had a higher frequency of polyps than other UC patients $(\mathrm{P}=0.001)$. For the $\mathrm{CD}$ patients, those with a higher ESR and platelet count had a higher incidence of strictures $(\mathrm{P} \leq 0.001)$ (Figure $4 B$ ). Similarly, we demonstrated that a possible combination of a higher ESR and lower eosinophil count indicated a higher incidence of fistula than other combinations $(\mathrm{P} \leq 0.001)$ (Figure $4 C$ ). Our data therefore suggested that the combination of two routine clinical parameters increased their predictive accuracy, which could be an additional tool in identifying IBD patients at risk for complications. 
Table 4 Predictive factors for stricture in Crohn's disease in univariate and multivariate logistic regression analyses

\begin{tabular}{|c|c|c|c|c|c|c|c|c|}
\hline Variable & Category & Patient number & \multicolumn{3}{|c|}{ Univariate analysis } & \multicolumn{3}{|c|}{ Multivariate analysis } \\
\hline Age & $<40 / \geq 40$ y & $319 / 120$ & 0.497 & $0.287-0.861$ & $0.013^{*}$ & 0.466 & $0.262-0.829$ & $0.009^{*}$ \\
\hline Sex & Male/female & $151 / 288$ & 1.225 & $0.767-1.958$ & 0.395 & 0.867 & $0.520-1.446$ & 0.584 \\
\hline Stage & Severe/others & $123 / 316$ & 0.522 & $0.304-0.896$ & $0.018^{*}$ & 0.524 & $0.301-0.912$ & $0.022^{*}$ \\
\hline ESR & $<18.5 / \geq 18.5$ & $259 / 180$ & 0.423 & $0.261-0.685$ & $0.000^{*}$ & 0.500 & $0.298-0.840$ & $0.009^{*}$ \\
\hline PLT & $<328 \times 10^{9} / \geq 328 \times 10^{9}$ & $313 / 126$ & 0.457 & $0.265-0.790$ & $0.005^{*}$ & 0.521 & $0.290-0.937$ & $0.029^{*}$ \\
\hline
\end{tabular}

*, $\mathrm{P}<0.05$ was considered significant. $\mathrm{P}$ values were calculated by univariate and multivariate binary logistic regression analysis. $\mathrm{Cl}$, confidence interval; OR, odds ratio; ESR, serum erythrocyte sedimentation rate; PLT, platelet.
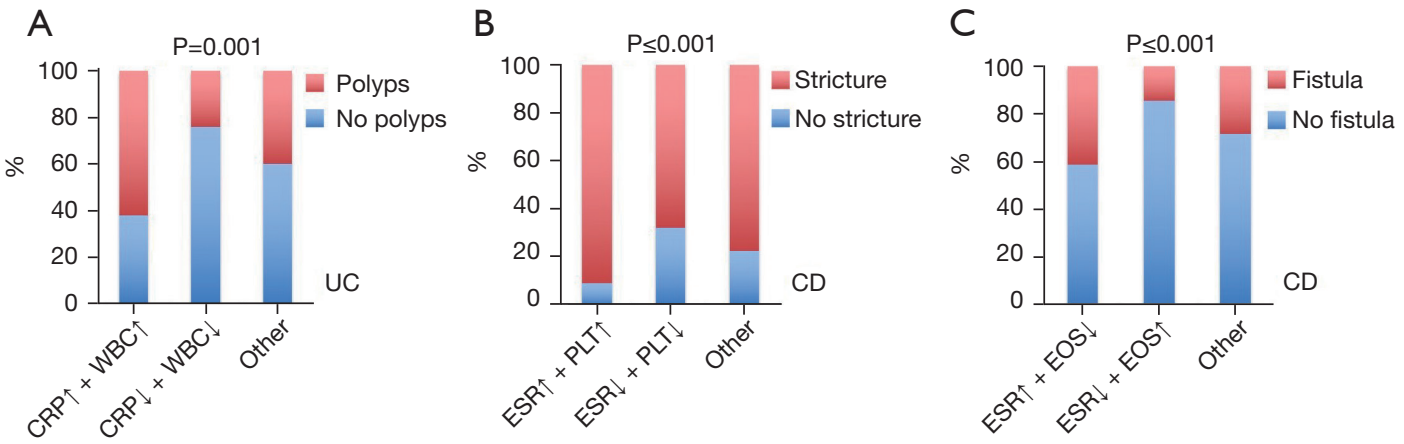

Figure 4 Dual parameter prediction system verification. (A) Patients with UC and both a high CRP level and a high WBC had a higher frequency of polyps than other UC patients $(\mathrm{P}=0.001)$; (B) for patients with $\mathrm{CD}$, those with both a higher ESR and higher PLT count had a higher incidence of strictures $(\mathrm{P} \leq 0.001)$; (C) CD patients with the combination of a higher ESR and a lower eosinophil count had a higher incidence of fistulas than those with other combinations $(\mathrm{P} \leq 0.001)$. UC, ulcerative colitis; CRP, C-reactive protein; WBC, white blood cell; CD, Crohn's disease; ESR, erythrocyte sedimentation rate; PLT, platelet.

\section{Discussion}

IBD significantly affects and impairs patients' quality of life, such as access to health care, education, employment and relationships. Several treatments are currently available for treating IBD patients, primarily including aminosalicylates, systemic corticosteroids, topical corticosteroids, antibiotics and immunomodulators. With limited efficacy, no cure is currently available, moreover, patients with IBD also frequently suffer from extra complications such as perforation, fistula, pseudopolyp formation, stenosis or cancer, which aggravates the burden and worries of IBD patients. Such outcomes are very difficult to treat once they develop, due to a lack of specific drugs, often require surgery or endoscopic treatment, commonly recur after surgery, and dramatically affect the quality of life of patients
$(27,28)$. Although many of these untoward complications may, in theory, be mitigated by screening patients, given the heterogeneous nature of IBD, therapeutic requirements vary considerably, and we have limited capacity to economically predict disease progression for individual patients despite years of investigation (29). Accordingly, the identification of inexpensive and reproducible clinical markers to stratify patients for individualisation of therapy in IBD should be an ongoing process because it may influence important therapeutic outcomes.

As we all know, cytokines are key mediators of cellular immune response in the intestine and can be used in new potential predictor and therapeutic targets for chronic intestinal inflammation complications, more biological therapies that target different molecular pathways aside 
from TNF blockade have been developed, such as those targeting Interleukin (IL)-6, IL-12/IL-23 pathway and IL-17. Although cytokines can reflect immune activity to a certain extent, more entries need to be detected and the price is relatively expensive, not all medical facilities are equipped for testing. A routine blood examination is one of the most rapid, convenient and inexpensive clinical examinations that can reflect a patient's inflammatory status and other blood conditions.

To our knowledge, WBC count, CRP level, and ESR, despite being highly sensitive but not very specific, are the most commonly used indicators to establish the diagnosis and assess disease activity during routine clinical workup for IBD patients. However, their use as biomarkers for IBD complications has a number of considerations, because an enhanced level of CRP or ESR has also been reported in patients with other inflammatory conditions beyond the colon $(19,30,31)$. In this study, we investigated whether blood tests routinely used for IBD patients in the clinical setting could predict disease-related complications, defined as the occurrence of fistulas, strictures and pseudopolyps. We found that a higher serum CRP level and WBC count determined at diagnosis independently indicated a higher hazard for the earlier occurrence of pseudopolyp formation in UC patients, and the predictive effect was stronger if both indicators increased simultaneously. Moreover, many lines of research have clearly demonstrated that PLTs are potent immune modulators and effectors, and may play a role in the pathogenesis of IBD (20,32-34). We demonstrated that higher serum ESR and PLT counts were associated with CD patients' strictures, which is consistent with the results of previous similar studies $(35,36)$. Of note, we also demonstrated that higher serum ESR combined with decreased eosinophil count might indicate more specifically CD patients who may progress to fistula. To date, a number of studies have suggested that eosinophils contribute to the pathogenesis of gastrointestinal inflammation due to a broad range of biologically active mediators, including antimicrobial, pro-remodelling or anti-inflammatory factors released by eosinophils $(37,38)$. Increased numbers of mucosal eosinophils in IBD have also been investigated; for instance, eosinophil activation in tissues could participate in early mucosal damage and disrupt the mucosal barrier $(39,40)$. However, there are no available studies concerning such an analysis, and therefore our findings may assist in predicting IBD complications more accurately.

The limitation of the present study was the small number of participants, so the results may not be relevant to the general population. However, these promising findings should be validated in a multicentre study.

\section{Conclusions}

A comprehensive evaluation of routine clinical parameters of complete blood counts was performed in IBD patients. Serum CRP level, ESR, WBC count, PLT count and eosinophil count appear to be promising predictors of IBD complications. These biomarkers have the advantage of being routinely available, non-invasive and low-cost, which will benefit patients both clinically (rational application of biological agents and immunosuppressants; minimizing the sequelae of complications) and financially.

\section{Acknowledgments}

Funding: This study was supported by the National Natural Science Foundation of China (No. 81800491).

\section{Footnote}

Reporting Checklist: The authors have completed the STROBE reporting checklist. Available at https://atm. amegroups.com/article/view/10.21037/atm-22-123/rc

Data Sharing Statement: Available at https://atm.amegroups. com/article/view/10.21037/atm-22-123/dss

Conflicts of Interest: All authors have completed the ICMJE uniform disclosure form (available at https://atm. amegroups.com/article/view/10.21037/atm-22-123/coif). The authors have no conflicts of interest to declare.

Ethical Statement: The authors are accountable for all aspects of the work in ensuring that questions related to the accuracy or integrity of any part of the work are appropriately investigated and resolved. All procedures performed in this study involving human participants were in accordance with the Declaration of Helsinki (as revised in 2013). The study was approved by the Ethics Committee at Ruijin Hospital Affiliated to Shanghai Jiao Tong University School of Medicine (approval ID:2014-Clinical Ethics Review No.16a). Individual consent for this retrospective analysis was waived.

Open Access Statement: This is an Open Access article distributed in accordance with the Creative Commons 
Attribution-NonCommercial-NoDerivs 4.0 International License (CC BY-NC-ND 4.0), which permits the noncommercial replication and distribution of the article with the strict proviso that no changes or edits are made and the original work is properly cited (including links to both the formal publication through the relevant DOI and the license). See: https://creativecommons.org/licenses/by-nc-nd/4.0/.

\section{References}

1. Torres J, Mehandru S, Colombel JF, et al. Crohn's disease. Lancet 2017;389:1741-55.

2. Ordás I, Eckmann L, Talamini $M$, et al. Ulcerative colitis. Lancet 2012;380:1606-19.

3. Peyrin-Biroulet L, Loftus EV Jr, Colombel JF, et al. The natural history of adult Crohn's disease in populationbased cohorts. Am J Gastroenterol 2010;105:289-97.

4. Thia KT, Sandborn WJ, Harmsen WS, et al. Risk factors associated with progression to intestinal complications of Crohn's disease in a population-based cohort. Gastroenterology 2010;139:1147-55.

5. Khanna R, Bressler B, Levesque BG, et al. Early combined immunosuppression for the management of Crohn's disease (REACT): a cluster randomised controlled trial. Lancet 2015;386:1825-34.

6. Schreiber S, Reinisch W, Colombel JF, et al. Subgroup analysis of the placebo-controlled CHARM trial: increased remission rates through 3 years for adalimumab-treated patients with early Crohn's disease. J Crohns Colitis 2013;7:213-21.

7. Rieder F, Latella G, Magro F, et al. European Crohn's and Colitis Organisation Topical Review on Prediction, Diagnosis and Management of Fibrostenosing Crohn's Disease. J Crohns Colitis 2016;10:873-85.

8. Hugot JP, Chamaillard $M$, Zouali $\mathrm{H}$, et al. Association of NOD2 leucine-rich repeat variants with susceptibility to Crohn's disease. Nature 2001;411:599-603.

9. Huang H, Fang M, Jostins L, et al. Fine-mapping inflammatory bowel disease loci to single-variant resolution. Nature 2017;547:173-8.

10. Luo Y, de Lange KM, Jostins L, et al. Exploring the genetic architecture of inflammatory bowel disease by whole-genome sequencing identifies association at ADCY7. Nat Genet 2017;49:186-92.

11. Jostins L, Ripke S, Weersma RK, et al. Host-microbe interactions have shaped the genetic architecture of inflammatory bowel disease. Nature 2012;491:119-24.

12. Liu JZ, van Sommeren S, Huang H, et al. Association analyses identify 38 susceptibility loci for inflammatory bowel disease and highlight shared genetic risk across populations. Nat Genet 2015;47:979-86.

13. Essers JB, Lee JJ, Kugathasan S, et al. Established genetic risk factors do not distinguish early and later onset Crohn's disease. Inflamm Bowel Dis 2009;15:1508-14.

14. Rivas MA, Beaudoin M, Gardet A, et al. Deep resequencing of GWAS loci identifies independent rare variants associated with inflammatory bowel disease. Nat Genet 2011;43:1066-73.

15. Howell KJ, Kraiczy J, Nayak KM, et al. DNA Methylation and Transcription Patterns in Intestinal Epithelial Cells From Pediatric Patients With Inflammatory Bowel Diseases Differentiate Disease Subtypes and Associate With Outcome. Gastroenterology 2018;154:585-98.

16. McGovern DP, Kugathasan S, Cho JH. Genetics of Inflammatory Bowel Diseases. Gastroenterology 2015;149:1163-1176.e2.

17. Levine A, Sigall Boneh R, Wine E. Evolving role of diet in the pathogenesis and treatment of inflammatory bowel diseases. Gut 2018;67:1726-38.

18. Osada T, Ohkusa T, Okayasu I, et al. Correlations among total colonoscopic findings, clinical symptoms, and laboratory markers in ulcerative colitis. J Gastroenterol Hepatol 2008;23 Suppl 2:S262-S267.

19. Alper A, Zhang L, Pashankar DS. Correlation of Erythrocyte Sedimentation Rate and C-Reactive Protein With Pediatric Inflammatory Bowel Disease Activity. J Pediatr Gastroenterol Nutr 2017;65:e25-7.

20. Ripoche J. Blood platelets and inflammation: their relationship with liver and digestive diseases. Clin Res Hepatol Gastroenterol 2011;35:353-7.

21. Binder MT, Becker E, Wiendl M, et al. Similar Inhibition of Dynamic Adhesion of Lymphocytes From IBD Patients to MAdCAM-1 by Vedolizumab and Etrolizumab-s. Inflamm Bowel Dis 2018;24:1237-50.

22. Iwata M, Hirakiyama A, Eshima $Y$, et al. Retinoic acid imprints gut-homing specificity on T cells. Immunity 2004;21:527-38.

23. Danese S, Panés J. Development of drugs to target interactions between leukocytes and endothelial cells and treatment algorithms for inflammatory bowel diseases. Gastroenterology 2014;147:981-9.

24. Park SC, Jeen YT. Anti-integrin therapy for inflammatory bowel disease. World J Gastroenterol 2018;24:1868-80.

25. Sands BE. Leukocyte Anti-Trafficking Strategies: Current Status and Future Directions. Dig Dis 2017;35:13-20.

26. Politis DS, Katsanos KH, Tsianos EV, et al. Pseudopolyps 
in inflammatory bowel diseases: Have we learned enough? World J Gastroenterol 2017;23:1541-51.

27. Lönnfors S, Vermeire S, Greco M, et al. IBD and healthrelated quality of life -- discovering the true impact. J Crohns Colitis 2014;8:1281-6.

28. Manninen P, Karvonen AL, Huhtala H, et al. Mortality in ulcerative colitis and Crohn's disease. A population-based study in Finland. J Crohns Colitis 2012;6:524-8.

29. Panes J, Jairath V, Levesque BG. Advances in Use of Endoscopy, Radiology, and Biomarkers to Monitor Inflammatory Bowel Diseases. Gastroenterology 2017;152:362-373.e3.

30. Kathiresan S, Larson MG, Vasan RS, et al. Contribution of clinical correlates and 13 C-reactive protein gene polymorphisms to interindividual variability in serum C-reactive protein level. Circulation 2006;113:1415-23.

31. Lewis JD. The utility of biomarkers in the diagnosis and therapy of inflammatory bowel disease. Gastroenterology 2011;140:1817-1826.e2.

32. Senchenkova E, Seifert H, Granger DN. Hypercoagulability and Platelet Abnormalities in Inflammatory Bowel Disease. Semin Thromb Hemost 2015;41:582-9.

33. Jenne CN, Kubes P. Platelets in inflammation and infection. Platelets 2015;26:286-92.

Cite this article as: Li T, Qian Y, Bai T, Li J. Prediction of complications in inflammatory bowel disease using routine blood parameters at diagnosis. Ann Transl Med 2022;10(4):185. doi: 10.21037/atm-22-123
34. Yan SL, Russell J, Granger DN. Platelet activation and platelet-leukocyte aggregation elicited in experimental colitis are mediated by interleukin-6. Inflamm Bowel Dis 2014;20:353-62.

35. Stidham RW, Guentner AS, Ruma JL, et al. Intestinal Dilation and Platelet:Albumin Ratio Are Predictors of Surgery in Stricturing Small Bowel Crohn's Disease. Clin Gastroenterol Hepatol 2016;14:1112-1119.e2.

36. Higgins PD. Measurement of Fibrosis in Crohn's Disease Strictures with Imaging and Blood Biomarkers to Inform Clinical Decisions. Dig Dis 2017;35:32-7.

37. Rothenberg ME, Hogan SP. The eosinophil. Annu Rev Immunol 2006;24:147-74.

38. Hogan SP, Waddell A, Fulkerson PC. Eosinophils in infection and intestinal immunity. Curr Opin Gastroenterol 2013;29:7-14.

39. Wallon C, Persborn M, Jönsson M, et al. Eosinophils express muscarinic receptors and corticotropin-releasing factor to disrupt the mucosal barrier in ulcerative colitis. Gastroenterology 2011;140:1597-607.

40. Dubucquoi S, Janin A, Klein O, et al. Activated eosinophils and interleukin 5 expression in early recurrence of Crohn's disease. Gut 1995;37:242-6.

(English Language Editor: K. Brown) 